\title{
CARACTERÍSTICAS CLÍNICO EPIDEMIOLÓGICAS DE PACIENTES CON SÍNDROME CORONARIO AGUDO DE LA UNIDAD DE CUIDADOS INTENSIVOS DEL HOSPITAL FÉLIX TORREALVA GUTIÉRREZ. ICA, PERÚ
}

\author{
Jesús Milagrito Avalos-Cabrera ${ }^{1, a}$, Fernando Carranza-Quispe ${ }^{2, a}$ \\ 1. Médico Residente Medicina Intensiva, Hospital Regional de Ica \\ 2. Médico Asistente Hospital Regional de Ica \\ a. Facultad de Medicina Humana, Universidad de Ica, Perú.
}

\section{RESUMEN}

Objetivo: Determinar las características clínico epidemiológicas del Síndrome Coronario Agudo (SICA) en los pacientes hospitalizados en la Unidad de Cuidados Intensivos (UCI) del Hospital Félix Torrealva Gutierrez en el año 2010 . Material y métodos: Diseño descriptivo observacional, se evaluaron 34 historias clínicas, se evaluaron variables como edad, sexo, diagnóstico al ingreso, factores de riesgo coronario, complicaciones clínicas intrahospitalarias, etc. Los datos obtenidos se expusieron en tablas, se compararon los resultados con la literatura revisada. Resultados: Los resultados mostraron que el SICA, representó el $50 \%$ de las causas de ingreso en la $\mathrm{UCl}$,el $85,29 \%$ de los pacientes tuvieron 60 años o más, predominaron los varones $(73,53 \%)$ sobre las mujeres $(26,47 \%)$, la formas clínica que predominó fue el infarto agudo de miocardio $(67,64 \%)$, hubo predominio del sexo masculino en angina inestable como en el infarto agudo de miocardio, la mayoría de pacientes se hospitalizó 6 horas o más después de iniciado el cuadro clínico,el 61,77\% de los pacientes presentaban hipertensión arterial como factor de riesgo coronario,la Diabetes Mellitus y las Dislipidemias constituyeron el segundo y tercer factor de riesgo de importancia ( 26,47 y $23,52 \%$ ),las arritmias cardiacas fueron las complicaciones más frecuentes con $47,05 \%$, la tasa de mortalidad fue de $14,71 \%$. Conclusiones: La forma clínica predominante fue el infarto agudo de miocardio; los factores de riesgo coronarios más importantes fueron la hipertensión arterial (HTA), la Diabetes Mellitus y las Dislipidemias, las complicaciones clínicas intrahospitalarias más importantes fueron las arritmias cardíacas.

Palabras Clave: Síndrome Coronario Agudo, epidemiología, cardiología (fuente: DeCS BIREME)

\section{CLINICAL EPIDEMIOLOGICAL CHARACTERISTICS OF PATIENTS WITH ACUTE CORONARY SYNDROME IN THE INTENSIVE CARE UNIT OF THE FELIX TORREALVA GUTIERREZ HOSPITAL IN ICA, PERU.}

\begin{abstract}
Objective :To Determine the clinical epidemiological features of Acute Coronary Syndrome (SICA) in hospitalized patients in the Intensive Care Unit (ICU) of the Hospital Felix Torrealva Gutierrez in 2010. Material and methods: Design observational descriptive, were evaluated 34 clinical histories, were evaluated variables such as age, sex, diagnosis at admission, coronary risk factors, clinical complications hospital, etc. The data obtained are presented in tables, the results were compared with the literature reviewed. Results: The results showed that the SICA, accounted for $50 \%$ of the causes of admission to the ICU, the $85.29 \%$ of the patients were 60 years or more, there was a predominance of males $(73.53 \%)$ on women $(26.47)$, the forms clinic that prevailed was the acute myocardial infarction with $67.64 \%$, there was a predominance of males in unstable angina and acute myocardial infarction, themost patients are hospitalized 6 hours or more after the beginning of the clinical picture, the $61.77 \%$ of the patients had hypertension and coronary risk factor, diabetes mellitus and dyslipidemia were the second and third risk factor of importance ( 26.47 and $23.52 \%$ ), cardiac arrhythmias were the most frequent complications with $47.05 \%$, the mortality rate was $14.71 \%$.Conclusions: The prevailing clinical form was the acute myocardial infarction; coronary risk factors more important were the high blood pressure (hypertension), diabetes mellitus and dyslipidemia, hospital clinical complications were the most important cardiac arrhythmias.
\end{abstract}

Key words: Acute coronary syndrome, epidemiology, cardiology. (Source: MeSH NLM)

\section{INTRODUCCIÓN}

La Cardiopatía Isquémica, con su conocida alta incidencia, es en nuestros días una de las patologías más relevantes tanto en términos de mortalidad como de pérdida de calidad de vida en los países del mundo desarrollado y no desarrollados (2). El estudio Framingham, realizado en 1940 y que constituye la primera investigación epidemiológica longitudinal y prospectiva que analizó los factores de riesgo coronario, identificó, además de la edad y

el sexo, el tabaquismo, la hipertensión arterial, la hipercolesterolemia y la diabetes mellitus como los principales $(3,4)$. Las enfermedades cardiovasculares son, en la actualidad, la principal causa de muerte en los países industrializados y se espera que también lo sean en los países en vías de desarrollo en el año $2020^{(1,2)}$.

En los países de mayor alcance económico, a pesar del 
desarrollo en los medios diagnósticos y terapéuticos de que disponen, en la actualidad pocos de ellos reportan discreta disminución en las tasas de mortalidad por enfermedades cardiovasculares $^{(3,4)}$. En Estados Unidos en el año 2003 según registros, 4.497.000 pacientes acudieron a los departamentos de emergencia con el diagnóstico primario de enfermedad cardiovascular ${ }^{(5)}$.

En la Unión Europea las enfermedades cardiovasculares fueron la causa directa de muerte de más de 1.9 millones de personas en el año 2000 , lo que representa el $43 \%$ de todas las muertes de cualquier edad en hombres y el $55 \%$ en las mujeres ${ }^{(6)}$.

Estos padecimientos son también frecuentes en los países en vías de desarrollo; en ellos los adultos mayores son especialmente vulnerables a las enfermedades crónicas no transmisibles. Además en estos países las personas tienden a desarrollar enfermedades a edades más tempranas, sufrirlas durante más tiempo y fallecer antes que en los países de alto ingreso, se calcula que las enfermedades cardiovasculares reducen la expectativa de vida 7 años ${ }^{(8)}$.

Perú no es ajeno a esta problemática de salud, en el año 2008 las enfermedades cardiovasculares ocuparon el tercer lugar como causa de morbilidad y el segundo en mortalidad $^{(10)}$.

Dentro del espectro de las enfermedades cardiovasculares merece especial atención el Síndrome Coronario Agudo (SICA), por ser la manifestación más frecuente y temible de la cardiopatía isquémica y porque aporta el mayor número de defunciones en las personas afectadas.

El conocimiento actual de la fisiopatología del síndrome coronario nos plantea un reto en lo que se refiere al tratamiento precoz sobre todo en las salas de emergencia, de coronarias o en las de cuidados intensivos.

Por todo lo anteriormente expuesto, el objetivo del estudio fue estudiar el comportamiento y el tratamiento de los pacientes con Síndrome Coronario Agudo (SICA) en una Unidad de Cuidados Intensivos (UCl).

\section{MATERIAL Y MÉTODOS}

El diseño del estudio fue de tipo retrospectivo, observacional, descriptivo, transversal, el nivel de investigación es básico, aplicativo.

El universo estuvo formado por todos los pacientes que acudieron al hospital Félix Torrealva Gutiérrez el año 2010.

La población estuvo conformada por todos los pacientes atendidos en el servicio de Cuidados Intensivos durante el año 2010 y la muestra por todos los pacientes hospitalizados durante el año 2010 en la Unidad de Cuidados Intensivos con el diagnóstico de Síndrome Coronario Agudo.

Criterios de inclusión: Pacientes adultos con diagnóstico clínico de Síndrome Coronario Agudo (SICA) que fueron diagnosticados con clínica, EKG y enzimas, cuyas historias contaron con los datos del cuestionario aplicado, tratados en la UCI del Hospital Félix Torrealva Gutiérrez.
Criterios de exclusión:Se excluyeron las historias clínicas que no tuvieron los criterios de diagnóstico de síndrome coronario agudo y los registros correspondientes a las variables a estudiar.

Para la recolección de los datos se usó un cuestionario adhoc. Los grupos de edades se evaluaron en 4 etapas, el paciente adulto joven (menores de 40 años), el paciente adulto (40 a 59 años) el paciente de la tercera edad (60 a 74 años) y el grupo de 75 años o más. La clasificación en los 3 tipos de síndrome coronario agudo se hizo siguiendo los lineamientos de las clasificaciones más actualizadas. Los factores de riesgo coronario y las complicaciones se reflejaron en frecuencias.

La mortalidad se obtuvo del cociente del total de fallecidos dividido entre el total de pacientes ingresados con el diagnóstico de Síndrome Coronario Agudo (SICA) multiplicado por 100 .

Se tomó en cuenta los siguientes aspectos: confidencialidad, anonimato en la revisión de las historias clínicas.

Para el procesamiento y análisis de datos se utilizó el programa Microsoft Office Excel versión 2007, para la base de datos y análisis de los mismos.

\section{RESULTADOS}

Se estudiaron 34 historias que cumplian con los criterios de inclusión establecidos para la presente investigación.

El síndrome coronario agudo, dentro de las enfermedades cardiovasculares, representó el $50 \%$ de las causas de ingreso en la $\mathrm{UCl}$, o sea de cada 10 pacientes que ingresaron por alguna patología cardiovascular 5 tenían una afección coronaria aguda.

El 85,29\% de los pacientes fueron mayores de 60 años, predominaron los hombres $(73,53 \%)$ sobre las mujeres ingresadas con este diagnóstico $(26,47 \%)$.

La forma clínica que predominó fue el infarto agudo de miocardio con 67,64\%, el 32,36 \% de los pacientes presentó angina inestable, en ambas formas clínicas predominaron los pacientes mayores de 60 años.

En relación al sexo hubo predomino del masculino tanto en angina inestable $(26,47 \%$ vs $5,89 \%)$ como en infarto agudo de miocardio (47,05 vs $20,59 \%)$.

La estratificación del Síndrome Coronario Agudo (SICA) con y sin elevación del segmento ST mostró que la mayor afectación ocurrió en los pacientes del sexo masculino para todos los grupos de edad, con excepción de los pacientes del grupo 40 a 59 años donde hubo predominio del sexo femenino a favor de los pacientes con SICAEST. De los 23 casos con infarto de miocardio, 16 (69,5\%) tuvieron el diagnostico de infarto con elevación del segmento ST 
mientras que $7 \quad(30,4 \%)$ fueron catalogados como IMA sin elevación del segmento ST.

El análisis del tiempo de evolución mostró que en el caso de la angina inestable el 11,76\% tuvo una evolución d 6 a 12 horas, el $8,82 \%$ menos de 6 horas y el 5,88\% entre 12 y 24 horas o más de 24 horas respectivamente. Los pacientes con infarto agudo con ST elevado el 14,71\% llegó entre 12 y 24 horas, el 11,76 \% más de 24 horas y menos de 6 horas, finalmente en los pacientes con infarto de miocardio ST no elevado el $5,8 \%$ se presentó en igual porcentaje entre 6 y 12 horas, 12 y 24 horas y más de 24 horas.

El $61,77 \% \%$ de los pacientes presentaban hipertensión arterial (HTA) como factor de riesgo coronario más frecuente, la Diabetes Mellitus y las Dislipidemias constituyen el segundo y tercer factor de riesgo de importancia en el grupo estudiado ( $26,47 \%$ y $23,52 \%$ ).

El $44,1 \%$ no tenían antecedentes de enfermedad cardiológica previa al ingreso, el antecedente presente que predominó fue Insuficiencia cardiaca $(20,58 \%)$. El $14,71 \%$ de los pacientes de la investigación eran portadores de arritmias y el 5,88\% presentaron miocardiopatía.

El $50 \%$ de los pacientes no presentaban historia isquémica previa, el $38,2 \%$ habían sufrido de un síndrome coronario agudo (SICA) .

El $35,29 \%$ de los pacientes no sufrieron ninguna de las complicaciones clínicas evaluadas, las complicaciones más frecuentes fueron: con 47,05\%, falla ventricular izquierda expresada por la insuficiencia cardiaca con $38,23 \%$, angina post infarto se presentó en $20,59 \%$ de los casos, el $11,76 \%$ desarrollaron edema agudo pulmonar, la mortalidad se presentó en $14,71 \%$ de los casos ingresados.

En relación al tratamiento trombolítico, la estreptocinasa fue aplicada al $30,43 \%$ de los pacientes con IAMEST y no se aplicó este tratamiento al $69,57 \%$ de ellos. Con relación a la topografía, la trombolisis se aplicó con mayor frecuencia en la localización anterior $(21,74 \%)$, mientras que en la localización inferior se aplicó en $8,7 \%$ de los casos.

De 23 pacientes con infarto agudo de miocardio 16 $(69,57 \%)$ no recibieron trombolisis, las causas más frecuentes por la que no se aplicó el tratamiento fueron: el tiempo transcurrido desde el inicio de los síntomas $>12$ horas, la edad mayor de 75 años y la inestabilidad hemodinámica identificada por los signos de insuficiencia cardiaca en el momento del ingreso, esto se observó con mayor frecuencia en las mujeres.

El ácido acetilsalicílico fue el fármaco que con mayor frecuencia se prescribió en estos pacientes $(67,65 \%)$, le siguieron en orden de frecuencia los inhibidores de la enzima de conversión de angiotensina (IECA) con $61,76 \%$, la nitroglicerina intravenosa $(55,8 \%)$, la furosemida en 55,88 $\%$, la heparina $(50,23 \%)$.

Los factores que más se relacionaron en frecuencia, con la mortalidad de los pacientes atendidos en la unidad de terapia intensiva con un síndrome coronario agudo fueron: el Infarto agudo de miocardio con elevación del ST el cual se presentó en el $75,0 \%$ del total de fallecidos, localización inferior $22 \%$, la edad $\geq$ a 65 años de edad (100\%), el shock cardiogénico $(100 \%)$, la neumonía $36 \%$ y la insuficiencia cardiaca $27 \%$.

Tabla 1. Síndrome coronario, distribución etaria.

\begin{tabular}{lcccc}
\hline \multirow{2}{*}{ Edad (años) } & \multicolumn{2}{c}{ Hombres } & \multicolumn{2}{c}{ Mujeres } \\
\cline { 2 - 5 } & $\mathbf{N}^{\circ}$ & $\%$ & $\mathbf{N}^{\circ}$ & $\%$ \\
\hline$<$ de $\mathbf{4 0}$ & - & - & - & - \\
$\mathbf{4 0}-\mathbf{5 9}$ & 3 & 8,82 & 2 & 5,89 \\
$\mathbf{6 0}-\mathbf{7 4}$ & 18 & 52,9 & 7 & 20,59 \\
& & 4 & & \\
$\mathbf{7 5}$ a mas & 4 & 11,7 & - & - \\
& & 6 & & \\
Total & 25 & 73,5 & 9 & 26,47 \\
\hline
\end{tabular}

Tabla 2. Síndrome coronario, formas clínicas según edad.

\begin{tabular}{cccccccc}
\hline \multirow{2}{*}{$\begin{array}{c}\text { Formas } \\
\text { Clínicas }\end{array}$} & \multicolumn{2}{c}{$\begin{array}{c}40- \\
59 a n ̃ o s\end{array}$} & \multicolumn{2}{c}{$\begin{array}{c}60-74 \\
\text { años }\end{array}$} & \multicolumn{2}{c}{75 a mas } \\
\cline { 2 - 8 } & $\mathbf{N}^{\circ}$ & $\%$ & $\mathbf{N}^{\circ}$ & $\%$ & $\mathbf{N}^{\circ}$ & $\%$ \\
\hline $\begin{array}{c}\text { Angina inestable } \\
\text { Infarto agudo de } \\
\text { miocardio }\end{array}$ & 2 & 5,88 & 8 & 23,52 & 1 & 2,94 \\
\hline
\end{tabular}

Tabla 3. Síndrome coronario: Complicaciones clínicas.

\begin{tabular}{lcc}
\hline \multirow{2}{*}{\begin{tabular}{c} 
Complicaciones \\
\multicolumn{1}{c}{ clínicas }
\end{tabular}} & $\mathbf{2}$ Total \\
\cline { 2 - 3 } & & $\%$ \\
\hline Ninguna & 12 & 35,29 \\
Arritmias cardiacas & 16 & 47,05 \\
Insuficiencia cardiaca & 13 & 38,23 \\
Edema Agudo & 3 & 8,82 \\
Pulmonar & 7 & 20,59 \\
Angina Post Infarto & 4 & 11,76 \\
Shock cardiogenico & 5 & 14,71 \\
Muerte & & \\
\hline
\end{tabular}

Tabla 4. Síndrome coronario: Factores de riesgo

\begin{tabular}{ccccccc}
\hline & \multicolumn{9}{c}{ Sexo } \\
$\begin{array}{c}\text { Factores de } \\
\text { riesgo }\end{array}$ & \multicolumn{9}{c}{ Masculino } & \multicolumn{2}{c}{ Femenino } & \multicolumn{2}{c}{ Total } \\
\cline { 2 - 7 } & $\mathbf{N}$ & \% & $\mathbf{N}$ & $\%$ & $\mathbf{N}$ & $\%$ \\
\hline Fumador & 6 & 17.65 & 1 & 2.94 & 7 & 20.59 \\
Ex fumador & 4 & 11.76 & - & & 4 & 11.76 \\
Dislipidemia & 5 & 14.71 & 3 & 8.82 & 8 & 23.52 \\
Diabetes & 5 & 14.71 & 4 & 11.76 & 9 & 26.47 \\
Mellitus & 4 & 11.76 & 2 & 5.88 & 6 & 17.65 \\
Obesidad & 16 & 47.04 & 5 & 14.73 & 21 & 61.77 \\
HTA & & & & &
\end{tabular}




\section{DISCUSIÒN}

El síndrome coronario agudo es en la actualidad un importante problema de salud en los países desarrollados y también en los países en vías de desarrollo, donde existe además un incremento significativo ${ }^{(5,6)}$. En Estados Unidos se reportó un número elevado de egresos de pacientes con este diagnóstico en el año 2001, estimándose que el $30 \%$ de ellos tuvieron un infarto agudo de miocardio con elevación de ST ${ }^{(17)}$ En este estudio, coincidiendo con lo reportado en la literatura, hubo una mayor frecuencia de ingreso de pacientes con síndrome coronario agudo con relación a otras causas de ingreso por enfermedades cardiovasculares. Según García ${ }^{(1)}$, Jiménez ${ }^{(4)}$ y Permanyer (16) en sus estudios predominaron los pacientes con 60 años y más. Existe una relación epidemiológicamente demostrada entre la enfermedad y el envejecimiento, esto no quiere decir que todas las enfermedades aumentan con la edad.

Las diferencias sexuales en la morbilidad por Cardiopatía Isquémica están bien documentadas. Se han avanzado hipótesis ambientales y genéticas para explicar estas diferencias, pero su importancia relativa sigue sin estar clara. Se cree que los factores hormonales desempeñan un papel importante. Sin embargo, los porcentajes de morbilidad por cardiopatía isquémica tanto en hombres como en mujeres varían sustancialmente de unos países a otros, y con el tiempo, lo que indica que también intervienen efectos ambientales. Como ha sido señalado por varios autores los pacientes del sexo masculino se ven con frecuencia más afectados con el síndrome coronario agudo en todas sus formas de presentación, con relación al sexo femenino. Las mujeres cuando se presentan con SCAEST lo hacen en edades más avanzadas que los hombres según lo reportado, esto no se corresponde con los resultados encontrados en el presente estudio donde al contrario en mujeres de 40 a 59 años hubo mayor número de casos que en varones de la misma edad.

Las mujeres se presentan con síntomas de similar frecuencia, duración y carácter que los hombres pero tienen más síntomas equivalentes de angina (disnea o síntomas atípicos).

Desde los primeros reportes del Framingham Heart Study se encontró que el nivel de colesterol está fuertemente relacionado al desarrollo posterior de enfermedad coronaria en la población, lo mismo sucede con la diabetes mellitus.

Según Roca y colaboradores ${ }^{(18)}$, la Diabetes Mellitus es otro de los grandes factores de riesgo estudiados, además ella tiene una serie de características trombogénicas como son las alteraciones de la coagulación, rigidez eritrocitaria, aumento de la viscosidad sanguínea y mayor adhesividad de las plaquetas al endotelio dañado, además suele asociarse a otros factores como la hipertensión arterial (HTA) y dislipidemia ${ }^{(20)}$.

El riesgo de complicaciones de estos pacientes es muy variable. Al mes siguiente del episodio coronario el riesgo de infarto agudo de miocardio (IAM) no fatal en los pacientes con angina inestable oscila entre el 2 al $5 \%$ y el riesgo de mortalidad entre el 5 y el 15\%. La máxima incidencia de complicaciones se observa durante la primera semana, presentándose aproximadamente el $50-60 \%$ de los casos de muerte o infarto (equivalente a un 1\% de muerte o infarto por día). Entre la primera semana y el primer mes, otro $25-35 \%$ del total presenta muerte o infarto y, finalmente, el $15-20 \%$ de complicaciones aparecen en los siguientes 5 meses. Entre el 25 y el $50 \%$ de los pacientes dados de alta reingresaran durante este intervalo.

La mortalidad por infarto agudo de miocardio (IAM) es una función exponencial en relación con el tiempo, de modo que una proporción sustancial de la misma tiene lugar dentro de la primera hora y el $90 \%$, en las primeras $24 \mathrm{~h}$. La mayoría de los fallecimientos suceden antes de que el paciente tenga la oportunidad de llegar al hospital, de modo que la reducción de la mortalidad hospitalaria sólo representa una pequeña fracción de la mortalidad total. Las Arritmias Cardíacas son el mecanismo de la mayor parte de los fallecimientos que se producen en las primeras horas del infarto agudo de miocardio (IAM).

Los pacientes con shock cardiogénico representan el 11,76 $\%$ de los infarto agudo de miocardio admitidos en el hospital y su mortalidad es muy elevada (> 70\%), no modificada por el tratamiento fibrinolítico. No obstante a los resultados adversos en los pacientes con esta complicación referidos anteriormente, se observó que la mortalidad $(14,71 \%)$ en los pacientes con Síndrome Coronario Agudo (SCA) en el periodo de estudio fue comparable a otros estudios en centros de otro nivel de atención.

Está demostrado que la mayor reducción de la mortalidad con la terapia fibrinolítica se alcanza cuando ésta es aplicada en las primera horas desde el inicio de los síntomas. Adicionalmente los pacientes con infarto anterior, bloqueo de rama izquierda presumiblemente nuevo y área de riesgo de mayor tamaño dado por el número de derivaciones afectadas y la intensidad de la desviación del segmento ST, son los que obtienen mayor beneficio con la terapia de reperfusión farmacológica. Uno de los mensajes más importantes transmitidos por el conjunto de datos disponibles es que el tratamiento fibrinolítico se infrautiliza en los pacientes con IAMEST.

Los factores del paciente relacionados con un intervalo más largo hasta la decisión de solicitar atención médica ha sido reportada en varios estudios y Braunwald señala entre estos la edad avanzada, el sexo femenino, los antecedentes de angina o diabetes entre otros. En este estudio el autor encontró varios factores en común, pero contrario a ello, los pacientes del sexo masculino mostraron mayor frecuencia en la demora de solicitud de atención médica.El tratamiento de reperfusión farmacológica no se aplica según diversos estudios a una proporción significativa $(25 \%$ - $30 \%)$ de pacientes con infarto con elevación del segmento ST. Desafortunadamente a pesar de que la población estudio fue pequeña, la falta de aplicación de estreptocinasa $(69,5 \%)$ en el estudio fue mayor a la reportada por algunos autores.

En el registro GRACE (Global Registry of Acute Coronary Events) ${ }^{(30)}$, que enrolo 2084 pacientes durante las primeras 12 horas tras un infarto con elevación del segmento ST, no 
se aplicó al 30\% de los pacientes con criterios para ello. Los factores que más mostraron correlación con la falta de aplicación fueron los antecedentes de injerto vascular arterial, de diabetes y de insuficiencia cardiaca congestiva, así como la edad superior a 75 años.

La terapia antiplaquetaria con ácido acetilsalicílico según lo revisado en la literatura, es el fármaco que con mayor frecuencia se indicó en los pacientes con Síndrome Coronario Agudo (SICA), proporcionando beneficios en la reducción de la mortalidad y en la disminución de las tasas de episodios vasculares. Por la evidencia ya existente se considera que estos beneficios deben estar presentes también en los pacientes de este estudio. Debe señalarse que el registro GRACE, un estudio observacional amplio donde participaron 95 hospitales en 14 países de Europa, Asia y América reportó frecuencia mayores en el uso de aspirina, betabloqueantes y heparina. También se reporta elevada frecuencia en el uso de IECA.

Los anticoagulantes se utilizan en el Síndrome Coronario Agudo sin elevación del ST (SCASEST) para inhibir la generación y/o actividad de la trombina y de esta forma reducir los episodios relacionados con la formación de trombos. Hay evidencia clara de que la anticoagulación es efectiva junto a la inhibición plaquetaria y que la combinación de las dos es más efectiva que cualquiera de ellas por separado. La utilización de heparina en el estudio fue baja con relación a estudios publicados.

Los factores que más se relacionaron en frecuencia, con la mortalidad de los pacientes atendidos en la unidad de terapia intensiva con un síndrome coronario agudo fueron: el Infarto agudo de miocardio con elevación del ST el cual se presentó en el $75.0 \%$ del total de fallecidos, localización inferior $22 \%$, la edad $\geq$ a 65 años de edad (100\%), el shock cardiogénico (100\%), la neumonía $36 \%$ y la insuficiencia cardiaca $27 \%$.

Se concluye que el comportamiento del síndrome coronario agudo manejado en la $\mathrm{UCl}$ del hospital Félix Torrealva Gutierrez de ESSALUD no difiere significativamente de lo reportado en la literatura.

Fuentes de financiamiento: autofinanciado.

Conflicto de intereses: los autores declaran no tener conflictos de interés.

\section{Correspondencia:}

Jesús Milagrito Avalos-Cabrera

Correo electrónico:milagritoac@hotmail.com

\section{REFERENCIAS BIBLIOGRAFICAS}

1. García-Castrillo L, Alonso Valle $H$, Suárez Antelo J, Soler W, Martín F, Balaguer $\mathbf{J}$ et al. Diagnóstico precoz del síndrome coronario agudo en los servicios de urgencias. Papel de la h-FABP. Emergencias 2004;16(1):233-8.

2. The GRACE Investigator. GRACE (Global Registry of Acute Coronary Events): a multinational registry of patients hospitalized with acute coronary syndromes. Am Heart J 2001; 141(2): 190-9.

3. Murga $\mathbf{N}$, Barrás $X$, Barrios $V$, Pedreira $M$. Actualización en cardiología clínica. Rev Esp Cardiol. 2008; 61 (Supl 1): 86-96.

4. Jiménez $\mathbf{M}$ y Gómez $\mathbf{M}$. Criterios terapéuticos en el síndrome coronario agudo Unidad Coronaria. Servicio de Cardiología. Rev Clin Esp 2003;203(2):83-5.

5. Guarda E et al. Insuficiente control de los factores de riesgo en prevención secundaria cardiovascular. Rev Med Chile. 2005, 133(1): 1147 $-1152$.

6. Mora $\mathbf{G}$ et al. Características clínicas y electrocardiográficas de los pacientes que ingresan en una unidad de dolor torácico en el contexto de la nueva definición del Infarto Agudo del Miocardio. Rev colombiana de Cardiología. 2005,11(7): 333 .

7. Márquez $\mathbf{Y}$ et al. Morbimortalidad por infarto agudo de miocardio (IAM), estudio de un año junio 2003-junio 2004. Hospital Faustino Pérez, Matanzas. Revista médica eléctronica Matanzas 2006; 28(2). Disponible en: http://www.revmatanzas.sld.cu/revista\%20medica/ ano\%202006/vol2\%202006/revista\%20medica $\% 20$ vol2\%202006.htm

8. Castillo E. Comportamiento clínico epidemiológico de la Cardiopatía Isquémica en la Unidad de Cuidados Intensivos polivalente. Rev Cubana Enfermer. 2008; 24(1):6-10.

9. Montes de Oca $\mathbf{S}$. Comportamiento del infarto agudo de miocardio (IAM) en el municipio Pedro María Ureña. [Trabajo para optar por el título de máster en Urgencias Médicas] Táchira: CDI Pedro María Ureña. 2006. 56 pp.

10. Moreno D. Síndrome Coronario Agudo. Comportamiento clínico - epidemiológico en Cuidados Intensivos [Trabajo para optar por el título de Máster en Urgencias Médicas] Maracaibo: CDI Panamericano. 2008. 64 pp.

11. Bueno $\mathbf{H}$ y col. Manejo del síndrome coronario agudo sin elevación del segmento ST en España. Estudio DESCARTES (Descripción del Estado de los Síndromes Coronarios Agudos en un Registro Temporal Español) Rev Esp Cardiol. 2005; 58(3):244-52.

12. Aranda $\mathbf{E}$ y col. Choque cardiogénico en síndrome coronario agudo: causas, criterios diagnósticos, tratamiento y mortalidad en el Instituto Nacional del Corazón en Lima- Perú Rev Med Hered. 2008;19(4):138-144.

13. Rubiera $\mathbf{R}$ y col. Síndrome coronario agudo. Caracterización clínico epidemiológica. Revista Cubana de Medicina Intensiva y Emergencias. 2009; 8(3): 1439-1449.

14. Braunwald E. Guidelines for the Management of Patients With Unstable Angina and Non-STSegment Elevation Myocardial Infarction. J Am Coll Cardiol. 2000; 36(1):970-1056

15. Terol I, San Martin MA, García A y col.Síndrome Coronario Agudo: nuevo enfoque fisiopatológico. Cardiovascular Risk Factors. 2003; 12(1):35-42. . 
16. Permanyer-Miralda G, Brotons C, Cascant P, Ribera A, Moral I, Soler-Soler J. Pacientes con síndrome coronario agudo: abordaje terapéutico (patrones de manejo) y pronóstico al año en un hospital terciario. Rev Esp Cardiol 1998; 51(12): 954-964

17. Anderson JL, Adams CD, Antman EM, Bridges CR, Calif RM, Casey DE, et al. Guidelines for the Management of Patients with Angina/Non -ST Elevation Myocardial Infarction. JACC. 2007; 50(7):1-157.

18. Davies MJ. The pathophysiology of acute coronary syndromes. Heart. 2000; 83(3):361-6.

19. Libby $\mathbf{P}$. Current concepts of the pathogenesis of the acute coronary syndromes. Circulation. 2001;104(3):365-72.

20. Alpert JS, Thygesen K, Antman EM, Bassand JP. Myocardial infarction redefined a consensus document of The Joint European Society/American
College of Cardiology Committee for redefinition of myocardial infarction.J Am Coll Cardiol. 2000;36(3):959-69.

21. Sánz G. El estudio PRIAMHO. Rev Esp Cardio. 1999; 52(1): 776-777.

22. Gutiérrez H. Comportamiento del Síndrome Coronario Agudo (SCA) en pacientes ingresados en la Terapia Intensiva. Revista Electrónica de PortalesMedicos.com 2010, 5(22).www.portalesmedicos.com/revista/vol05_n22 .htm (Publicado: 29/11/2010).

Recibido: 16 / 10/ 2012

Aceptado para publicación: 2112 / 2012 